\title{
Design and experimental evaluation of flextensional-cantilever based piezoelectric transducers for flow energy harvesting
}

Hyeong Jae Lee, Stewart Sherrit, Luis Phillipe Tosi, Tim Colonius

Hyeong Jae Lee, Stewart Sherrit, Luis Phillipe Tosi, Tim Colonius, "Design and experimental evaluation of flextensional-cantilever based piezoelectric transducers for flow energy harvesting," Proc. SPIE 9806, Smart Materials and Nondestructive Evaluation for Energy Systems 2016, 980610 (1 April 2016); doi: 10.1117/12.2219269 Health Monitoring, 2016, Las Vegas, Nevada, United States 


\title{
Design and Experimental Evaluation of Flextensional-Cantilever based Piezoelectric Transducers for Flow Energy Harvesting
}

\author{
Hyeong Jae Lee*a, Stewart Sherrit ${ }^{\mathrm{a}}$, Luis Phillpe Tosi ${ }^{\mathrm{b}}$, Tim Colonius ${ }^{\mathrm{b}}$ \\ a Jet Propulsion Laboratory, California Institute of Technology, Pasadena, CA 91109, USA; \\ ${ }^{\mathrm{b}}$ California Institute of Technology, Pasadena, CA 91109, USA Abstract
}

\begin{abstract}
Cantilever type piezoelectric harvesters, such as bimorphs, are typically used for vibration induced energy harvesting. However, a major drawback of a piezoelectric bimorph is its brittle nature in harsh environments, precipitating short life-times as well as output power degradation. The emphasis in this work is to design robust, highly efficient piezoelectric harvesters that are capable of generating electrical power in the milliwatt range. Various harvesters were modeled, designed and prototyped, and the flextensional actuator based harvester, where the metal cantilever is mounted and coupled between two flextensional actuators, was found to be a viable alternative to the cantilever type piezoelectric harvesters. Preliminary tests show that these devices equipped with $5 \times 5 \times 36 \mathrm{~mm}$ two piezoelectric PZT stacks can produce greater than $50 \mathrm{~mW}$ of power under air flow induced vibrations.
\end{abstract}

Keywords: Piezoelectric Devices, flow energy harvesting, transducers, flextensional, fluid structure interaction

\section{INTRODUCTION}

The promise of an "endless" energy source and maintenance-free hardware has made energy harvesting technologies an active research topic over the last decade. These traits enable the deployment of sensing and control to optimize systems in hard-to-reach and desolate locations. Among various transduction mechanisms, vibration-based piezoelectricity is an attractive avenue to energy harvesting due to its robustness (resistance to both a large range of temperatures and cyclic loading), as well as high power density and electromechanical coupling efficacy [1-4]. Bimorph-type piezoelectric harvesters have been studied primarily for their low transverse bending stiffness, which can yield large stresses with small amplitude forces relative to other piezoelectric actuators. When fluid kinetic energy is the target energy source, maximum output power is normally achieved when fluid-structure instabilities are exploited to strongly couple structural motion to flow forcing. Aeroelastic flutter, in particular, has been shown as an effective fluid-structure coupling mechanism when bimorph-type actuators have relatively low resonant frequencies (less than $300 \mathrm{~Hz}$ ). Yet a major drawback is their brittle nature when exposed to large deformation, resulting in short lifetimes [5, 6].

Flextensional actuators, in contrast, offer a higher energy conversion efficiency due to force amplification mechanisms present in their structural design. Since the piezoelectric material is always kept under a compressive load and the surrounding metal frame is designed to survive large amplitude vibration, their design offers a combination of both fatigue resistance and the high energy density in to piezoelectrics. Yet flextensional actuators are relatively stiff; thus, it is difficult to exploit aeroelastic flutter without additional modifications to the structure. One such modification is to augment the structure with a non-piezoelectric cantilever in a converging-diverging flow channel. Flutter may ensue when the cantilever is clamped between two flextensional actuators and extends downstream of the channel throat if the appropriate parameters are selected. The channel geometry, fluid and flow properties, along with cantilever geometry and material properties dictate the fluid-structure force amplitude and driving frequency at the cantilever's clamped end. One key advantage of this design is that the piezoelectrics can be completely isolated from the working/producing fluids, reducing the effects of corrosion, erosion, and overall degradation on the piezoelectric material [7].

This paper presents the preliminary results of the latter design. We are interested in energy harvesting to power systems deep in an oil well, where ambient pressures exceed $200 \mathrm{MPa}$ and temperatures $160{ }^{\circ} \mathrm{C}$. Ultimately, the overall power generated must be scalable to the order of Watts, likely via the aggregation of multiple flow energy harvesting sections. The flextensional/cantilever energy harvesters where designed and fabricated, and experimental results detailing their power output capabilities are presented here.

Smart Materials and Nondestructive Evaluation for Energy Systems 2016, edited by

Norbert G. Meyendorf, Theodoros E. Matikas, Kara J. Peters, Proc. of SPIE Vol. 9806, 980610

· (c) 2016 SPIE · CCC code: 0277-786X/16/\$18 · doi: 10.1117/12.2219269 


\section{CHARACTERIZATION OF PIEZOELECTRIC STACKS}

Piezoelectric stacks are composed of multiple thin, alternately poled, piezoelectric layers mechanically connected in series and electrically in parallel. They operate in what is known as 33 mode, where the applied force is parallel to the poling direction. The effective piezoelectric charge coefficient and capacitance of stacks are proportional to the number of the piezoelectric layers $n, d^{*}{ }_{33}=n \cdot d_{33}$ and $C^{*}=n^{2}$. $C$ respectively. This layering results in a high capacitance piezoelectric material that is better matched to electrical loads in comparison with a single piece piezoelectric element of the same dimensions. In order to design an effective flextensional/cantilever energy harvester, it is essential to characterize the properties of its piezoelectric stacks, which can be used for further analysis (i.e. FEA). This section briefly summarizes governing equations to characterize piezoelectric stacks.

The generated voltage of piezoelectric stack can be obtained using the piezoelectric constitutive equation, which is given by:

$$
\begin{aligned}
& S_{3}=s_{33}^{D} T_{3}+\mathrm{g}_{33} D_{3} \\
& E_{3}=-\mathrm{g}_{33} \cdot T_{3}+\beta_{33}^{T} \cdot D_{3},
\end{aligned}
$$

where $\mathrm{s}_{33}^{D}$ is the open-circuit elastic compliance, $g_{33}$ is piezoelectric voltage coefficient, $\beta_{33}^{T}$ is free dielectric impermeability constant, $S_{3}$ is the dimensionless strain, $T_{3}$ is the stress $\left(\mathrm{N} / \mathrm{m}^{2}\right)$ and $D_{3}$ is dielectric displacement or charge density $\left(\mathrm{C} / \mathrm{m}^{2}\right), \mathrm{E}_{3}$ is electric field $(\mathrm{V} / \mathrm{m})$.

When the electrodes are open-circuit $(D=0)$, the voltage developed across the piezoelectrics when the force is applied can be calculated as follows:

$$
V_{O C}=-\frac{d_{33} F}{C_{p}},
$$

where $C_{p}$ is the static capacitance of the piezoelectric material. Under the short-circuit condition, $E_{3}=0$, the charge produced by the piezoelectric element is as follows:

$$
Q=-d_{33} F
$$

The longitudinal strain under open circuit condition can be calculated from the constitutive equation.

$$
S_{3}=s_{33}^{D} T_{3},
$$

When an external capacitive load is connected to the piezoelectric harvester, the output voltage can be calculated as

$$
V_{\text {out }}=\frac{V_{O C}}{1+\frac{C_{L}}{C_{p}}}
$$

The maximum voltage that is delivered to the load can be achieved when the load capacitance $\left(C_{L}\right)$ is equal to the piezoelectric capacitance. The rms voltage $\left(V_{r m s}\right)$ and maximum average output power $\left(P_{\max , r m s}\right)$ from the piezoelectric element are then given by: 


$$
\begin{aligned}
& V_{r m s}=\frac{1}{\sqrt{2}} \frac{d_{33}}{\sqrt{\left(\frac{1}{\omega R_{L}}\right)^{2}+C_{p}^{2}}} F, \\
& P_{\text {max }, r m s}=\frac{\omega d_{33}^{2}}{4 C_{p}} F^{2},
\end{aligned}
$$

where $F$ is the exciting force, which is composed of a static force $\left(F_{0}\right)$ and a sinusoidal force $\left(F_{d}\right)$, (i.e., $\left.F=F_{0}+F_{d}\right)$.

For piezoelectric materials, we used commercially available piezoelectric stacks, which have a cross section of 5 $\mathrm{mm} \times 5 \mathrm{~mm}$ and a length of $36 \mathrm{~mm}$. Open circuit voltages of the tested stacks were investigated under static conditions. A schematic representation of the test setup is shown in Figure 1. The capacitance of the piezoelectric stack was found to be around $2.8 \mu \mathrm{F}$, which was measured at $1 \mathrm{kHz}$ using a network analyzer (HP 4294A, Hewlett Packard, Palo Alto, CA) under pre-stressed conditions. The voltage outputs of a piezoelectric stack under a force of $10 \mathrm{~N}$ every $10 \mathrm{sec}$ and a force of $10 \mathrm{~N}$ at $0.1 \mathrm{~Hz}$ are shown in Figure 2, showing the piezoelectric stack generates $0.9 \mathrm{~V}$. According to Eq. (2), the effective direct piezoelectric coefficient is found to be $0.25 \mu \mathrm{C} / \mathrm{N}$. The short-circuit elastic compliance of the stack, reciprocal of Young's modulus, can be determined by measuring the resonant frequency of the stack with Eq. (7), and is found to be $28 \mathrm{pm} / \mathrm{N}^{2}$.

$$
s_{33}^{E}=\frac{1}{4 \rho l^{2} f_{r}^{2}}=s_{33}^{D}+\frac{d_{33}^{2}}{\varepsilon_{33}^{T}}
$$

where $\rho, l, \mathrm{f}_{\mathrm{r}}$ and $\varepsilon^{\mathrm{T}}{ }_{33}$ are density, length, resonant frequency and permittivity of the piezoelectric material.

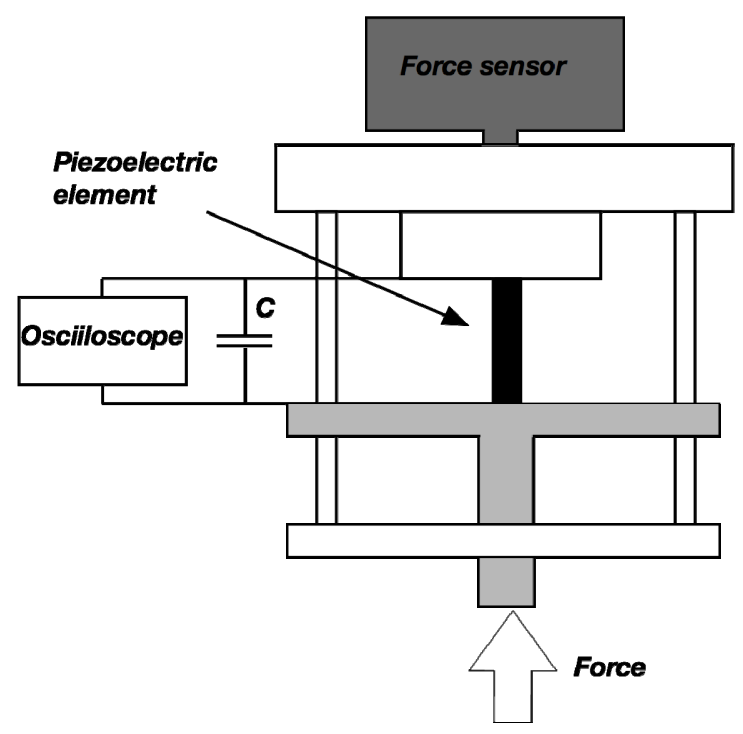

Figure 1: The experimental setup used for the evaluation of piezoelectric stacks. 

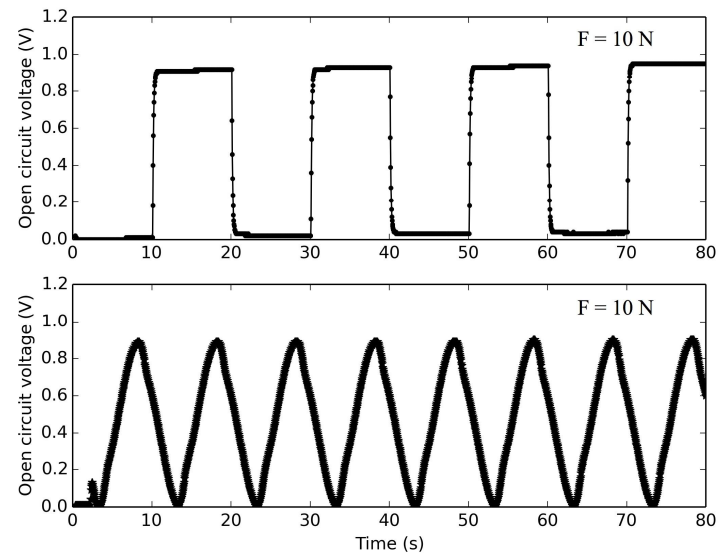

Figure 2: Measured piezoelectric open circuit voltages across the piezoelectric materials under static and quasi-static conditions as a function of time. The amplitude of applied forces for both conditions is $10 \mathrm{~N}$.

Figure 3 shows the theoretically calculated output power as a function of force on the piezoelectric stack based on the determined properties above, assuming that the resistive load is matched to the impedance of piezoelectric stacks in the range of $100 \mathrm{~Hz}$ to $500 \mathrm{~Hz}$. This is a typical flutter frequency range when the piezoelectric harvester undergoes fluid driven self-sustained oscillations. As shown, the piezoelectric stack can generate powers of milliwatts when more than $10 \mathrm{~N}$ of force is applied on the piezoelectric material. Considering that the flextensional actuator can amplify the fluid force by a factor ranging from 10 to 20 [9], flextensional/cantilever based harvesters can offer tens of milliwatts when an appropriate flow passage and cantilever design provides a frequency-matched, high amplitude fluid-structure forcing function.

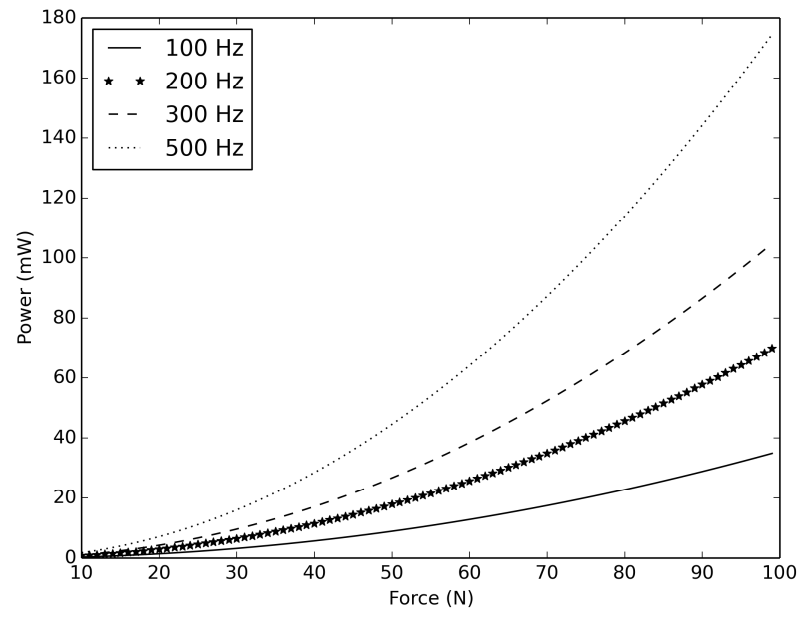

Figure 3: Calculated power output of piezoelectric stack as a function of force depending on the flutter frequency, where piezoelectric charge coefficient and static capacitance are $0.25 \mathrm{uC} / \mathrm{N}$ and $\mathrm{C}=2.8 \mathrm{uF}$, respectively.

\section{FLEXTENSIONAL/CANTILEVER HARVESTER DESIGN}

Figure 4 shows the schematic representation of a flow energy harvester based on the flextensional/cantilever harvester. The principle of operation is as follows: unsteady flow forces the exposed cantilever into self-sustained oscillations. The internal stresses to the cantilever due to the force distribution along its length and its dynamics are effectively countered 
by a point force and moment at its clamped end in the direction of the small axis of the flextensional frame. In the static/quasi-static sense, since the piezoelectric stacks are oriented in the horizontal direction, it can be seen that the mechanical advantage between the cantilever point force $F_{y}$ and the load on the flextensional $F_{x}$ is $F_{y} / F_{x} \propto x_{L} / y_{L}$, and the force magnitude on the flextensional is amplified if $y_{L}>x_{L}$.

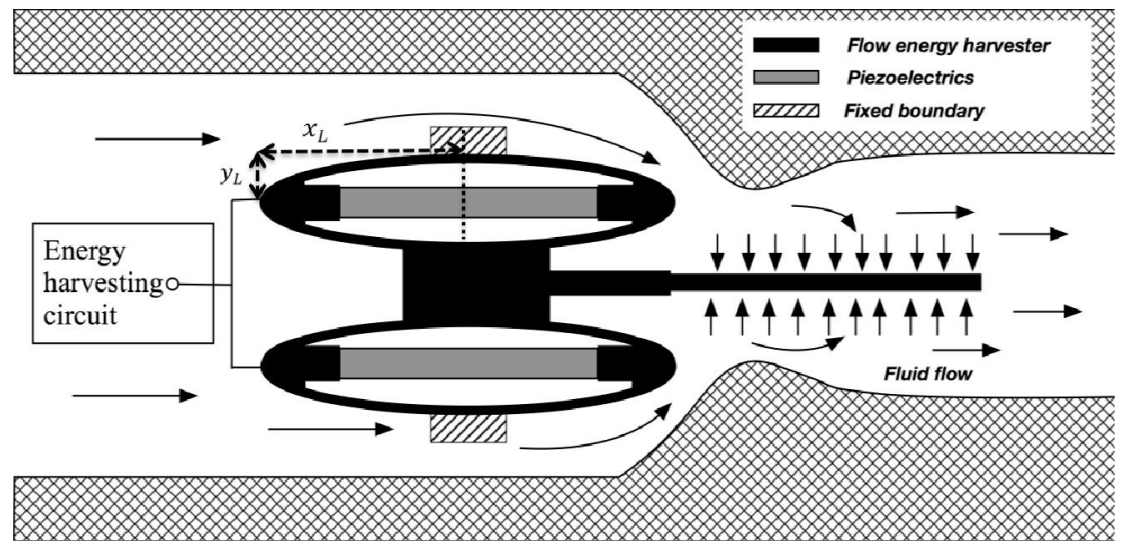

Figure 4: Schematic representation of an unibody flextensional/cantilever energy harvester. The arrows indicate the displacement directions.

A finite element model of the harvester was built using the commercial finite element software ABAQUS to analyze the effects of the design parameters on the resonant frequency of the flextensional/cantilever energy harvester. This frequency is the most important parameter for this energy harvesters since its matching to the frequency of the excitation maximizes the power harvested. The flextensional resonant frequencies need to be matched to those driven by the mounted cantilever, which couples to the fluid flow and provides the forcing function to the transducers. The dimension of the flextensional metal frame governs its resonant frequencies; thus, a frequency-matched flextensional system can be designed and fabricated by controlling the frames length and thickness. A modal analysis to predict the fundamental vibration modes and frequencies of the structure was carried out. Mode shape and frequency of the piezoelectric harvester design are shown in Figure 5. Note that since the resonant frequency of cantilever $(\sim 270 \mathrm{~Hz})$ is close to that of flextensional actuator $(\sim 340 \mathrm{~Hz})$, this design allows for the coupling between cantilever bending and flextural mode from flextensional when the flow induces flutter.
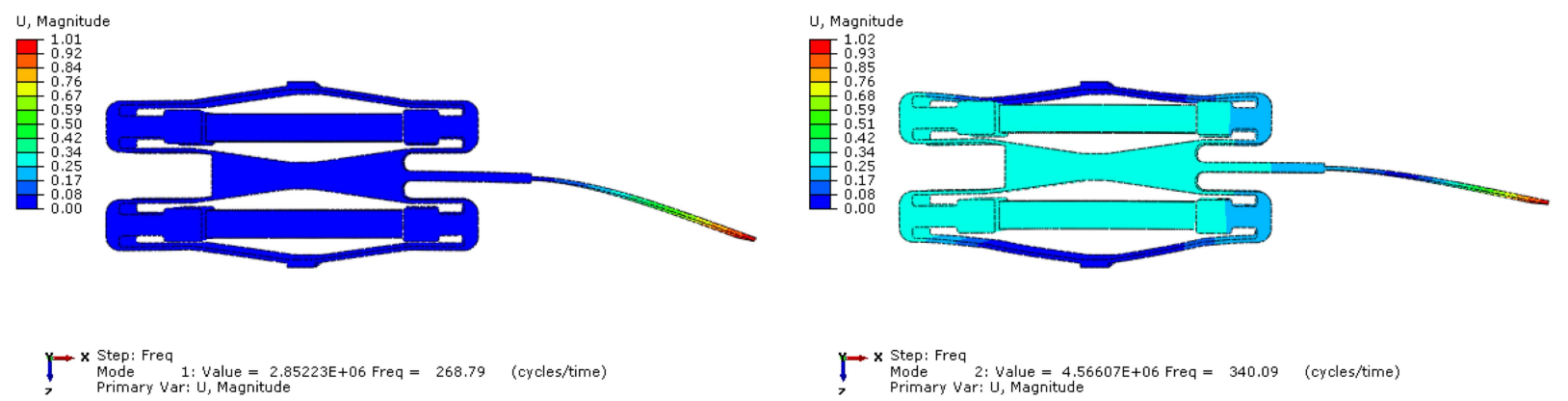

X Step: Freq
Mode $2:$ Value $=4.56607 \mathrm{E}+06$ Freq $=340.09 \quad$ (cycles/time)
Primary var: $U$, Magnitude

Figure 5: First and second modes of flextensional/cantilever energy harvester. The frequencies of first and second modes are $268 \mathrm{~Hz}$ and $340 \mathrm{~Hz}$, respectively.

\section{CHARACTERIZATION OF FLEXTENSIONAL/CANTILEVER HARVESTER}

A photo of the fabricated piezoelectric energy harvester is shown in Figure 6. In this design, we implemented a ball screw mechanism for pre-stressing, where the end caps produce axial loading on the piezoelectric stack. This ensures that the piezoelectric is always in compression during excitation and facilitates the assembly and integration of piezoelectric stacks onto the flextensional frame for repeatability. The measured electrical impedance from the harvester over a frequency range $(240-350 \mathrm{~Hz})$ is shown in Figure 7 . As expected, there are two distinct resonant frequencies are 
located around $275 \mathrm{~Hz}$ and $320 \mathrm{~Hz}$ that are related to the fundamental mode of the cantilever and flextensional actuator, respectively.

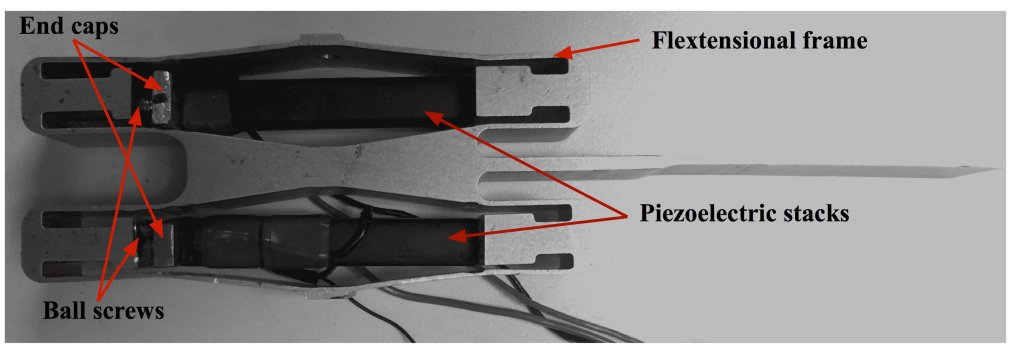

Figure 6: Photo of fabricated coupled mode flextensional/cantilever energy harvester.

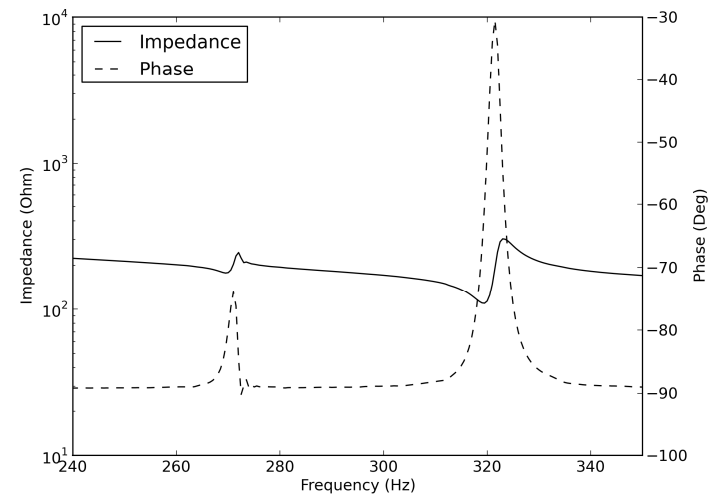

Figure 7: Measured electrical impedance (solid line) and phase (dashed line) of flextensional/cantilever energy harvester.

The performance of the flextensional/cantilever energy harvester was determined experimentally with nozzle integrated configuration under air flow. The generated output voltages were then recorded at the various flow rates. the voltage waveform from each piezoelectric stack across $100 \mathrm{Ohms}$ of resistance is shown in Figure 8 at air volumentric flow rate of $100 \mathrm{~L} / \mathrm{min}$. Voltage waveforms indicate that when one of the stack is under increasing compressive stress, the other is under decreasing compressive stress, leading to the 180 degrees phase difference. The measured $V_{r m s}=1.55$ $\mathrm{V}$.

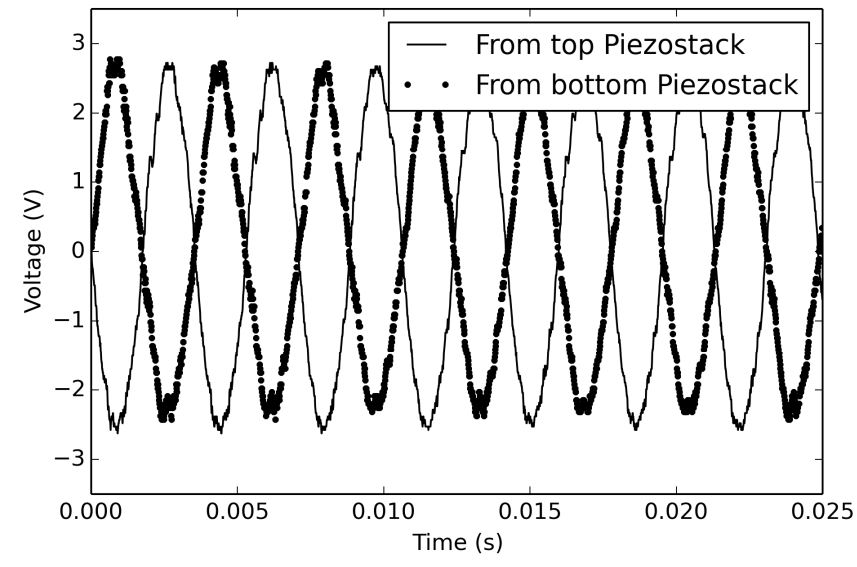

Figure 8: Voltage waveform of each piezoelectric stack from flextensional/cantilever energy harvester at a flow rate of 100 $\mathrm{L} / \mathrm{min}$. 
Figure 9 shows the corresponding power output from fabricated flextensional/cantilever actuator as a function of air flow rate. Experimental results showed that the energy harvester generated a maximum output power of $\sim 50 \mathrm{~mW}$ at $\sim 300 \mathrm{~Hz}$ under the airflow of $100 \mathrm{~L} / \mathrm{min}$. Corresponding frequencies were determined by applying fast Fourier transform (FFT) during measurements (plotted as a function of a flow rate). Note that the flutter frequencies of this harvester are between the resonant frequency of cantilever and flextensional actuator, exhibiting coupled modes when excited.
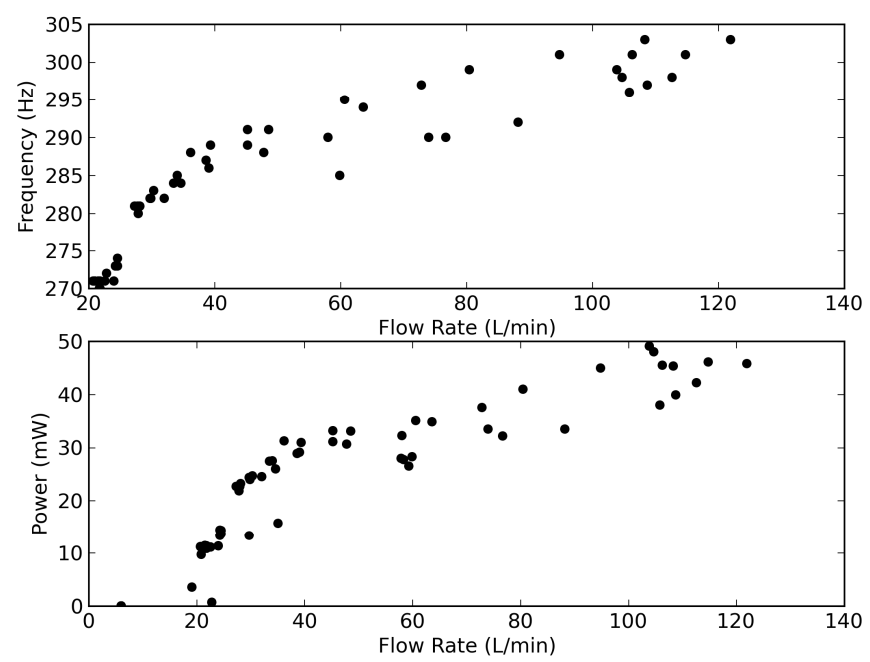

Figure 9: Exciting frequency and generated power from flextensional/cantilever energy harvester as a function of air flow rates.

The displacement amplitude of flextensional body was further investigated using high speed camera. Image data was taken as slow motion video (1200 fps) and processed frame by frame, as shown in Figure 10. The arrows on the figures indicate the measured location. Figure 11 shows the analyzed displacements from the flextensional/cantilever energy harvester at an air flow rate of $100 \mathrm{~L} / \mathrm{min}$ as a function of time. The frequency spectrum of the displacement profile was obtained by applying FFT, and is shown in the figure. The displacement amplitude of the flextensional body is found to be on the order of $\pm 0.2 \mathrm{~mm}$ in a vertical direction at around $300 \mathrm{~Hz}$.
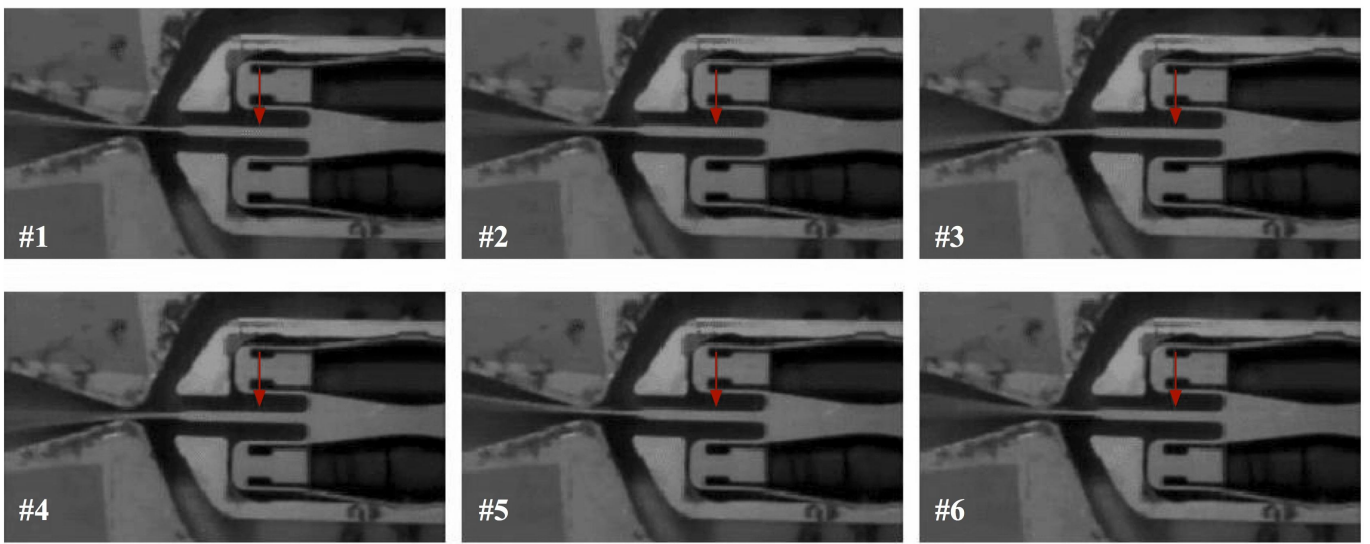

Figure 10: Snapshots of the motion of flextensional/cantilever energy harvester using high speed camera (Frame rate $=$ $1200 \mathrm{fps}$ ). The motion of the flextensional/cantilever energy harvester was tracked using object tracking algorithm, and the arrow indicates a location that measures the displacement. 

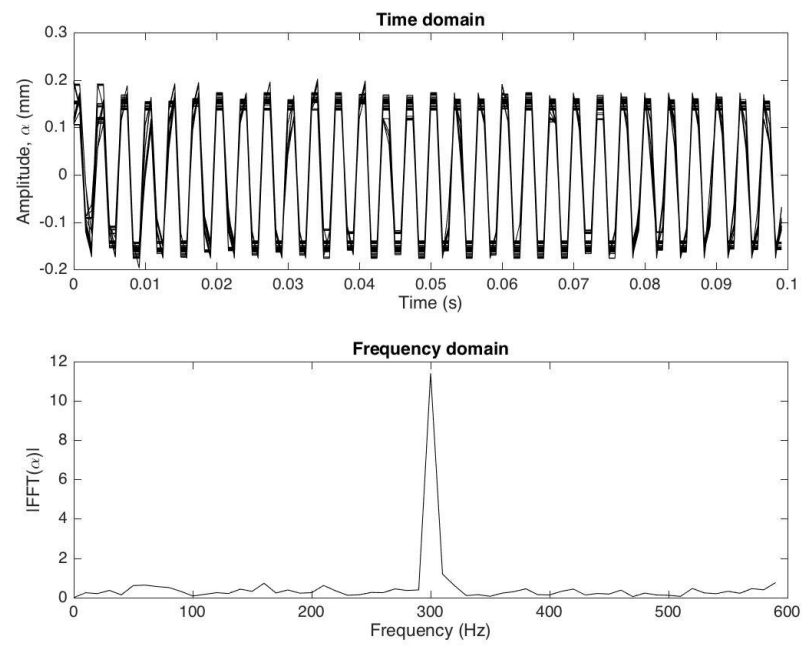

Figure 11: Video processed displacements and frequency of flextensional/cantilever energy harvester using object tracking algorithm.

\section{CONCLUSION}

We consider piezoelectric flow energy harvesting in an internal flow environment with the ultimate goal powering systems such as sensors in deep oil well applications. Flextensional/cantilever based energy harvesters produced $\approx 50$ $\mathrm{mW}$, which hits at a promising avenue of coupling tranduscers with fluid-flow. This power level is a significant step toward building a system design that can eventually deliver power in the Watts range to devices down within a well. Further research is currently underway to better understand and improve these and other flow energy harvester devices.

\section{REFERENCES}

[1] Roundy, S., Leland, E. S., Baker, J. et al., "Improving power output for vibration-based energy scavengers," IEEE Pervasive Computing, 4(1), 28-36 (2005).

[2] Roundy, S., "On the effectiveness of vibration-based energy harvesting," Journal of intelligent material systems and structures, 16(10), 809-823 (2005).

[3] Priya, S., "Advances in energy harvesting using low profile piezoelectric transducers," Journal of electroceramics, 19(1), 167-184 (2007).

[4] Platt, S. R., Farritor, S., and Haider, H., "On low-frequency electric power generation with PZT ceramics," IEEE/ASME Transactions on Mechatronics, 10(2), 240-252 (2005).

[5] Sherrit, S., Lee, H. J., Walkemeyer, P. et al., "Flow energy piezoelectric bimorph nozzle harvester."Proc. SPIE 9057, 90570D (2014).

[6] Sherrit, S., Lee, H. J., Walkemeyer, P. et al., "Fluid flow nozzle energy harvesters."Proc. SPIE 9435, 943507 (2015).

[7] Lee, H. J., Sherrit, S., Tosi, L. P. et al., "Piezoelectric Energy Harvesting in Internal Fluid Flow," Sensors, 15(10), 26039-26062 (2015). 